\title{
Sarcopenia after induction therapy is associated with reduced survival in patients undergoing esophagectomy for locally-advanced esophageal cancer
}

\author{
Connor J. Wakefield ${ }^{1} \wedge$, Fadi Hamati ${ }^{1}$, Justin M. Karush ${ }^{1}$, Andrew T. Arndt ${ }^{1}$, Nicole Geissen ${ }^{1}$, \\ Michael J. Liptay ${ }^{1}$, Jeffrey A. Borgia ${ }^{2}$, Sanjib Basu ${ }^{3}$, Christopher W. Seder ${ }^{1}$
}

${ }^{1}$ Rush University Medical Center, Department of Cardiovascular and Thoracic Surgery, Chicago, IL, USA; ${ }^{2}$ Rush University Medical Center, Departments of Pathology and Cell \& Molecular Medicine, Chicago, IL, USA; ${ }^{3}$ Rush University Medical Center, Department of Internal Medicine, Chicago, IL, USA

Contributions: (I) Conception and design: CJ Wakefield, CW Seder; (II) Administrative support: CJ Wakefield, F Hamati; (III) Provision of study materials or patients: CW Seder, JM Karush, AT Arndt, N Geissen, MJ Liptay; (IV) Collection and assembly of data: CJ Wakefield, F Hamati; (V) Data analysis and interpretation: CJ Wakefield, JA Borgia, S Basu; (VI) Manuscript writing: All authors; (VII) Final approval of manuscript: All authors.

Correspondence to: Christopher W. Seder, MD. Department of Cardiovascular and Thoracic Surgery, Rush University Medical Center; 1725 W. Harrison St, Suite 774, Chicago, IL 60612, USA. Email: Christopher_W_Seder@rush.edu.

\begin{abstract}
Background: The impact of sarcopenia on the outcome of esophageal cancer patients remains unknown in North American populations. The current study aims to investigate if sarcopenia at the time of esophagectomy for locally-advanced esophageal cancer (LAEC) is associated with survival.

Methods: Patients who underwent induction therapy followed by esophagectomy for LAEC between 2010-2018 at a single institution were identified. Exclusion criteria included follow-up less than 90 days and distant metastatic disease at the time of surgery. Demographic, treatment, and outcome data were retrospectively collected. Computed tomography (CT) scans following induction therapy were analyzed to calculate skeletal muscle index (SMI). Overall survival (OS) and disease-free survival (DFS) were examined using Kaplan-Meier and Cox Proportional Hazard regression analysis.

Results: Overall, 52 patients met inclusion criteria with a median BMI of 25 (IQR, 22.4-29.1) $\mathrm{kg} / \mathrm{m}^{2}$ and age of 65 (IQR, 57-70) years. Sarcopenia was present in 75\% (39/52) of patients at the time of surgery. Sarcopenic patients had a lower median BMI and higher median age when compared to non-sarcopenic patients. There was no difference in gender, race, stage, operative technique, post-operative complications, or hospital length of stay between sarcopenic and non-sarcopenic patients. With a median follow-up of 24.9 months, patients with sarcopenia at the time of esophagectomy had worse OS [median 24.3 (IQR, 9.9-34.5) vs. 50.9 (IQR, 25.6-50.9) months, $\mathrm{P}=0.0292$ ] and DFS [median 11.7 (IQR, 6.4-25.8) vs. 29.4 (IQR, 12.8-26.7) months, $\mathrm{P}=0.0387$ ] compared to non-sarcopenic patients.
\end{abstract}

Conclusions: Sarcopenia is associated with reduced overall and DFS in patients undergoing esophagectomy for LAEC.

Keywords: Locally-advanced esophageal cancer (LAEC); sarcopenia; esophagectomy

Submitted Aug 06, 2020. Accepted for publication Dec 10, 2020.

doi: $10.21037 /$ jtd-20-2608

View this article at: http://dx.doi.org/10.21037/jtd-20-2608

\footnotetext{
$\wedge$ ORCID: 0000-0003-3217-8121.
} 


\section{Introduction}

Esophageal cancer is the $8^{\text {th }}$ most common cancer diagnosis worldwide and carries a 5-year survival rate between $15-25 \%(1,2)$. Within the western hemisphere, esophageal cancer has increased in incidence faster than any other solid tumor in the last three decades (3). This rise has been primarily attributed to increased rates of obesity, gastroesophageal reflux disease (GERD), Barrett's esophagus, and associated esophageal adenocarcinoma (4).

Esophagectomy is a key component in the treatment of locally-advanced esophageal cancer (LAEC). However, esophagectomy carries with its high rates of morbidity and mortality, due to its invasive nature and the compromised functional status of patients at the time of surgery $(5,6)$. Esophageal cancer frequently leads to dysphagia, loss-ofappetite, and cancer-cachexia syndrome and induction chemoradiation (CRT) often exacerbates this impairment (7). Thus, identifying preoperative characteristics associated with poor long-term outcome may help determine which patients with LAEC benefit most from surgery after induction CRT and provide clinicians with data to guide preoperative optimization.

Sarcopenia, defined as a physiological state of reduced skeletal muscle mass, has been found to be associated with increased rates of postoperative complications and worse long-term outcomes in patients with a variety of gastrointestinal malignancies $(8,9)$. However, the impact of sarcopenia on the long-term outcomes of patients with LAEC who undergo esophagectomy remains controversial, especially in Western populations. We hypothesized that sarcopenic patients with LAEC who received induction CRT followed by surgery would have reduced overall and progression free survival when compared to non-sarcopenic patients. We present the following article in accordance with the STROBE reporting checklist (available at http:// dx.doi.org/10.21037/jtd-20-2608).

\section{Methods}

The study was conducted in accordance with the Declaration of Helsinki (as revised in 2013) and the Harmonized Tripartite Guideline for Good Clinical Practice from the International Conference on Harmonization. This study was reviewed and approved by the Rush University Medical Center Institutional Review Ethics Board (approval number 19100703-IRB01) and since it was retrospective in nature a waiver of the requirement for informed consent was included with approval.

An institutional database was used to identify all patients with LAEC that underwent induction CRT followed by esophagectomy between 2010-2018 at a single tertiary care medical center. Induction CRT regimens were administered at the discretion of the treating team in accordance with previously reported protocols $(10,11)$. Neoadjuvant radiation treatment consisted of 41.4-50 Gy external beam radiation administered in 25 to 28 fractions over a period of 5 weeks. Neoadjuvant chemotherapy was administered concurrently and included biweekly administration of 5 -fluorouracil in combination with platinum based agent for up to 6 cycles or weekly administration of carboplatin and paclitaxel for up to 6 cycles. All cases were discussed at a multidisciplinary tumor board prior to therapy to establish a treatment plan. Exclusion criteria included follow-up less than 90 days from surgery, presence of distant metastatic disease at surgery, and absence of radiographic imaging prior to surgery.

Variables collected included demographic, treatment, and outcome data. Sarcopenia was assessed by measuring skeletal muscle cross-sectional area at the $3^{\text {rd }}$ lumbar level using restaging computed tomography (CT) scans obtained at our institution. (Figure 1A,B). SliceOMatic v5.0 software (TomoVision, Magog, Canada) was utilized for body composition analysis and all CT scans were assessed by two operators trained on the software. All CT scans analyzed were performed after induction therapy and within 30-day of esophagectomy. Sarcopenia was defined using skeletal muscle index (SMI) cut-off values of $<41 \mathrm{~cm}^{2} / \mathrm{m}^{2}$ for females, $<43 \mathrm{~cm}^{2} / \mathrm{m}^{2}$ for males with a BMI $<25$, and $<53 \mathrm{~cm}^{2} / \mathrm{m}^{2}$ for males with a $\mathrm{BMI} \geq 25$, based on previous reports (12).

\section{Statistical analysis}

Baseline demographic and treatment data were compared between patients with sarcopenia and those without sarcopenia. Patients with missing data were excluded from analysis. Differences in baseline demographic and treatment data between the two groups were assessed with Student's $t$-test for parametric continuous variables and WilcoxonRanked Sum for non-parametric continuous variables and Chi-square tests and Fisher's Exact test for dichotomous and categorical variables. Interobserver correlation between the two operators who analyzed CT scans was assessed with Pearson correlation coefficient (r). Primary outcomes included overall survival (OS) and disease-free survival (DFS). Secondary outcomes included postoperative 

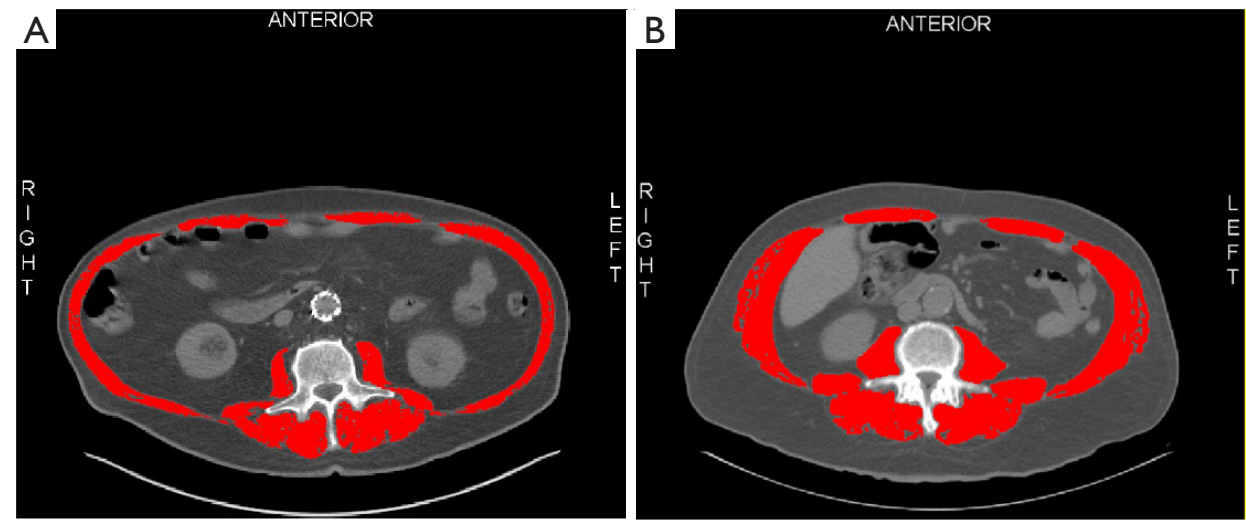

Figure 1 Pre-operative restaging CT scan of sarcopenic individual (A) and non-sarcopenic individual (B). Abdominal computed tomography scan at the $3^{\text {rd }}$ lumbar level with skeletal muscle cross-sectional area highlighted in red. (A) was found to be sarcopenic with a cross-sectional skeletal muscle area of $109.1 \mathrm{~cm}^{2}$, and skeletal muscle index of $33.56 \mathrm{~cm}^{2} / \mathrm{m}^{2}$. (B) Patient was found to be non-sarcopenic with a crosssectional skeletal muscle area of $112.9 \mathrm{~cm}^{2}$, and skeletal muscle index of $56.95 \mathrm{~cm}^{2} / \mathrm{m}^{2}$.

complications, hospital length-of-stay (LOS), and 30-day readmission rate. OS and DFS were examined using Kaplan-Meier survival analysis curves. Cox Proportional Hazard regression was utilized to perform adjusted survival analysis and to model which variables were independently associated with OS and DFS. Independent variables were selected for inclusion in the multivariate regression using stepwise selection and those found to have alpha $<0.1$ on univariate analysis or those previously reported in the literature to be significantly associated with esophagectomy outcomes were included in the Cox Proportional Hazard regression model. A proportional hazards assumption test was conducted for all Cox Proportional Hazard regression models utilizing Schoenfeld residuals. Two-sided statistical testing was utilized for all analyses and results with alpha $\leq 0.05$ was considered statistically significant for all analysis.

\section{Results}

Overall, 75 patients underwent induction CRT followed by esophagectomy for LAEC during the study period. Patients were excluded from analysis for not having retrievable CT imaging prior to esophagectomy $(\mathrm{n}=11)$, distant metastatic disease identified at the time of surgery $(n=5)$, or for being lost-to-follow-up $(n=7)$. There were no patients who expired in the immediate postoperative period. Thus, 52 patients met inclusion criteria with a median BMI of 25 (IQR, 22.4-29.1) $\mathrm{kg} / \mathrm{m}^{2}$ and age of 65 (IQR, 57-70) years (Table 1). Most patients were male [88\% (45/52)] and had adenocarcinoma [83\% (43/52)]. Sarcopenia was present in
$75 \%(39 / 52)$ of patients at the time of surgery. Sarcopenic patients had a median SMI of 38 (IQR, 34.6-42.1) $\mathrm{cm}^{2} / \mathrm{m}^{2}$ compared to 52.6 (IQR, 45.7-56.4) $\mathrm{cm}^{2} / \mathrm{m}^{2}$ for nonsarcopenic patients $(\mathrm{P}<0.001)$. Interobserver agreement between both operators on measurements of skeletal muscle area (SMA) was determined to be good with a Pearson coefficient of 0.87 . BMI was found to be significantly lower in the sarcopenic group, with a median value of 24.5 (IQR, $21.7-27.1) \mathrm{kg} / \mathrm{m}^{2}$ compared to 28.6 (IQR, 23.8-32.2) $\mathrm{kg} / \mathrm{m}^{2}$ in the non-sarcopenic group $(\mathrm{P}=0.025)$. Non-sarcopenia patients were also found to be significantly younger with a median age of 62 (IQR, 52-65) compared to sarcopenic patients who had a median age of 66 (IQR, 61-71). There was no difference in race, gender, cancer histology, stage, or operative approach between the two groups. Open operative approaches consisted of Ivor Lewis approach in 31 patients (60\%), McKeown (3-hole esophagectomy) in 7 patients (13\%), and transhiatal approach in 2 patients (4\%). Minimally invasive esophagectomy was performed in the remaining 12 patients (23\%). There was no difference in postoperative complications or hospital LOS between groups (Table 2). No continuous variables were made categorical throughout all analysis.

With a median follow-up of 24.9 months, patients with sarcopenia at the time of esophagectomy had worse OS [median 24.3 (IQR, 9.9-34.5) vs. 50.9 (IQR, 25.650.9) months, $\mathrm{P}=0.0292$ ] (Figure 2). Patients with sarcopenia at time of esophagectomy also had worse DFS [median 11.7 (IQR, 6.4-25.8) vs. 29.4 (IQR, 12.8-26.7) months, $\mathrm{P}=0.0387$ ] compared to non-sarcopenic patients (Figure 3). 
Table 1 Patient demographics

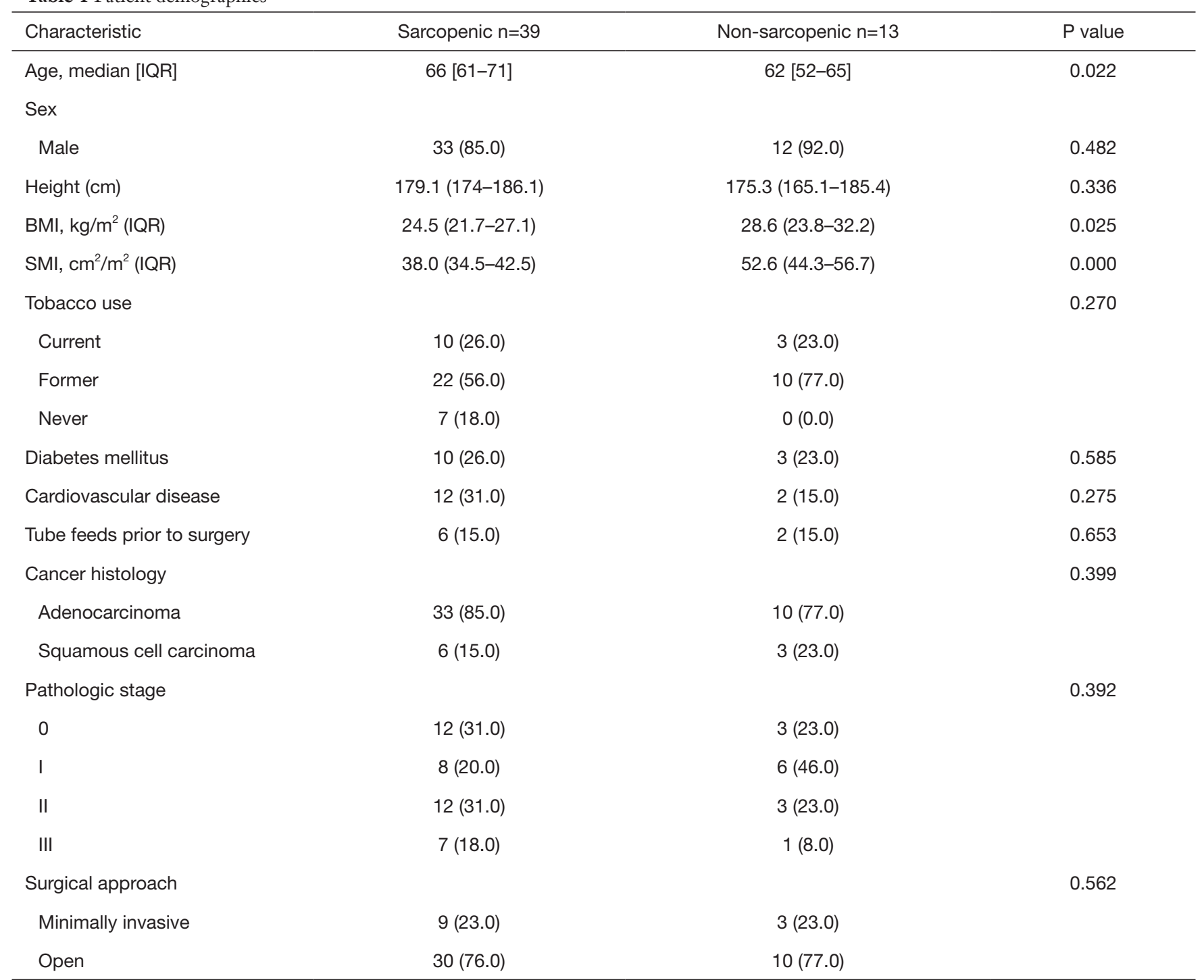

All data presented as $\mathrm{n}(\%)$, unless otherwise indicated. IQR, interquartile range; BMI, body mass index; SMI, skeletal muscle index.

Cox Proportional Hazard regression revealed sarcopenia was associated with worse OS when adjusted for by age, $\mathrm{BMI}$, presence of complication, and pathologic stage [hazard ratio $4.86,95 \%$ confidence interval $(1.41,16.77)$, $\mathrm{P}=0.012$ ] (Table 3). Both sarcopenia [hazard ratio $3.82,95 \%$ confidence interval $(1.09,13.37), \mathrm{P}=0.036]$ and pathologic stage III disease [hazard ratio $3.94,95 \%$ confidence interval (1.07, 14.53), $\mathrm{P}=0.039]$ were found to be associated with worse DFS on Cox Proportional hazard regression when adjusting for age, BMI, and presence of complications (Table 4).

\section{Discussion}

The effect of sarcopenia on long term outcomes in patients with LAEC remains controversial, with a paucity of data in Western populations. The current study suggests an association between preoperative sarcopenia and poor overall and DFS in patients with LAEC who underwent induction CRT followed by esophagectomy.

Reports are mixed on the effect sarcopenia has on longterm outcomes in patients who undergo esophagectomy for esophageal cancer (13-14). Two reports, one of 120 
Table 2 Hospital course and postoperative complications

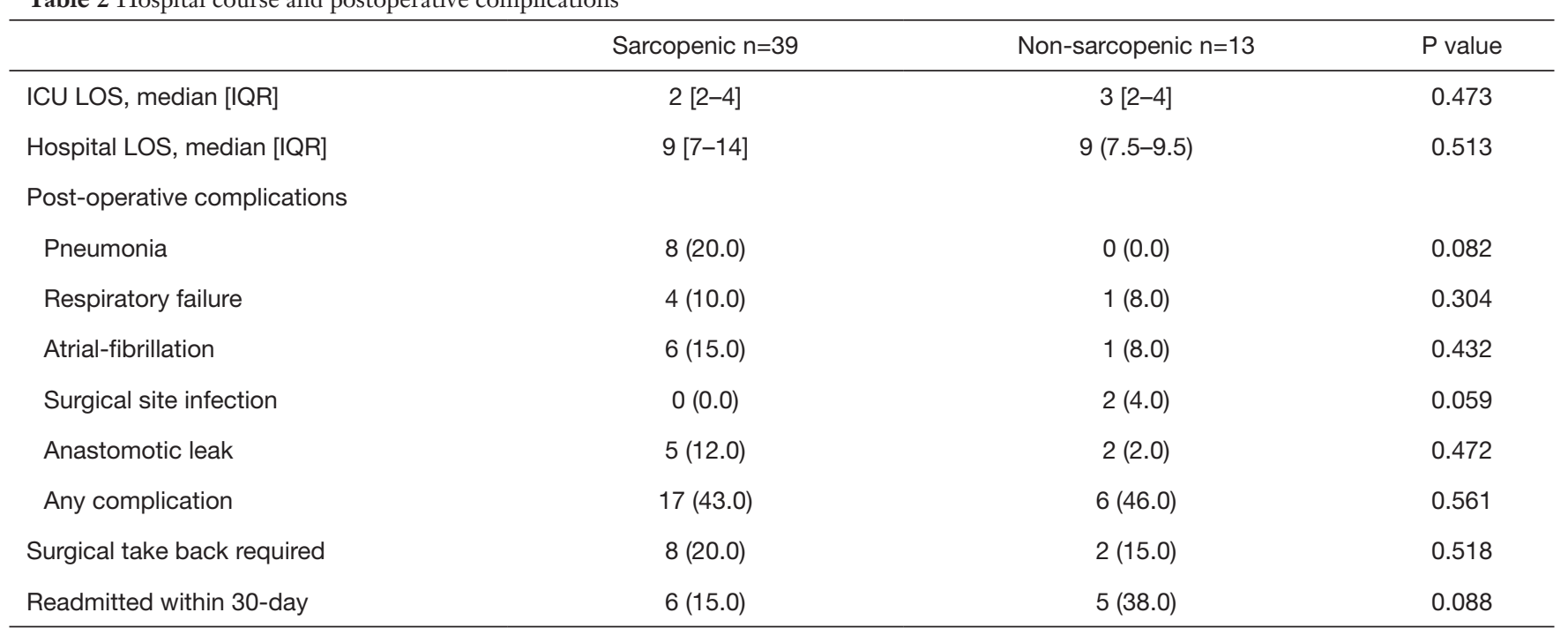

All data presented as $\mathrm{n}(\%)$, unless otherwise indicated. ICU, intensive care unit; LOS, length of stay; IQR, interquartile range.

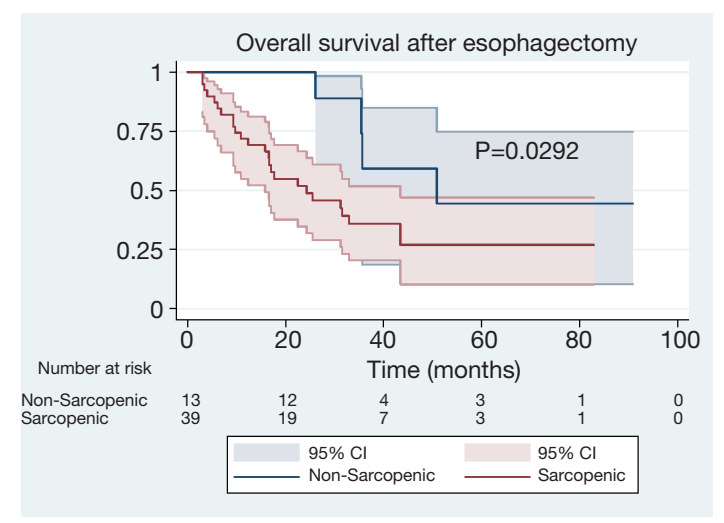

Figure 2 Kaplan-Meier curves for overall survival. Kaplan-Meier analysis of overall survival between patients with sarcopenia (red dotted line) and non-sarcopenic patients (blue solid line).

patients and the other of 173 patients who underwent esophagectomy for esophageal cancer found no difference in rates of morbidity, OS, or DFS between those with sarcopenia and those without $(13,18)$. However, both authors based their definition of sarcopenia off a study examining obese patient with respiratory or gastrointestinal malignancy (25). The current study used SMI cut-off values that account for gender and body mass index (BMI) (12). Considering that $52 \%$ of our cohort was obese at the time of operation, we felt it was important to stratify for obesity. The need to adopt sarcopenic cut-off values that account for BMI was further supported by the significant difference

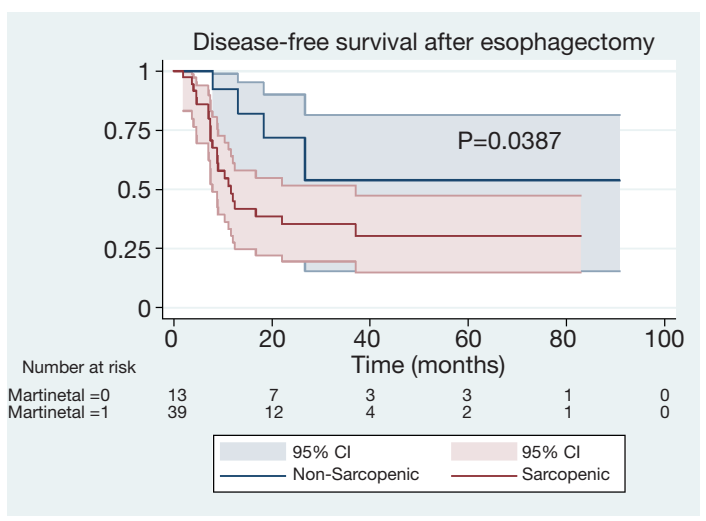

Figure 3 Kaplan-Meier curves for disease-free survival. Kaplan-Meier analysis of disease-free survival between patients with sarcopenia (red dotted line) and non-sarcopenic patients (blue solid line).

in median BMI between the sarcopenic and non-sarcopenic groups.

Other studies have found an association between sarcopenia and outcomes in esophageal cancer patients (15,21,26-28). One report on a large cohort of 230 patients, all who underwent induction CRT followed by transhiatal esophagectomy, found that sarcopenia was associated with both decreased OS and DFS (26). However, the authors used psoas muscle area (PMA) instead of total cross-sectional skeletal muscle area (SMA) when assessing for sarcopenia. Although PMA may offer some benefits, such as quicker assessment of sarcopenia, a recent study of patients with 
Table 3 Cox proportional hazard regression model for overall survival

\begin{tabular}{|c|c|c|c|c|}
\hline & Hazard ratio & Z score & $P$ value & $95 \% \mathrm{Cl}$ \\
\hline Age & 0.96 & -1.36 & 0.174 & {$[0.91,1.01]$} \\
\hline BMI & 1.06 & 1.22 & 0.221 & {$[0.97,1.16]$} \\
\hline \multicolumn{5}{|l|}{ Pathologic stage } \\
\hline II & 1.19 & 0.30 & 0.762 & {$[0.39,3.56]$} \\
\hline III & 1.05 & 0.08 & 0.939 & {$[0.27,4.08]$} \\
\hline Any complication & 1.14 & 0.28 & 0.781 & {$[0.46,2.78]$} \\
\hline
\end{tabular}

$\mathrm{Cl}$, confidence interval; BMI, body mass index.

Table 4 Cox proportional hazard regression model for disease-free survival

\begin{tabular}{|c|c|c|c|c|}
\hline & Hazard ratio & Z score & $P$ value & $95 \% \mathrm{Cl}$ \\
\hline Age & 1.00 & -0.12 & 0.901 & {$[0.94,1.05]$} \\
\hline BMI & 1.06 & 1.29 & 0.198 & {$[0.97,1.17]$} \\
\hline \multicolumn{5}{|l|}{ Pathologic stage } \\
\hline II & 1.38 & 0.52 & 0.602 & {$[0.39,3.56]$} \\
\hline III & 3.95 & 2.07 & 0.039 & {$[1.07,14.53]$} \\
\hline Any complication & 0.67 & -0.82 & 0.413 & {$[0.26,1.73]$} \\
\hline
\end{tabular}

$\mathrm{Cl}$, confidence interval; BMI, body mass index.

ovarian cancer demonstrated that PMA poorly correlates with SMA ( $\mathrm{r}=0.52)$ and is not associated with OS (29). It is not clear if calculating PMA or SMA is superior when assessing sarcopenia in patients with esophageal cancer. In addition, the current study included patients that underwent various surgical techniques in comparison to only the transhiatal esophagectomy mentioned in the previous study. Another report of 130 esophageal cancer patients who received induction chemotherapy followed by surgery found that patients with sarcopenia had worse OS and DFS (15). However, in this European study, only 5.4\% of patients received induction radiation therapy whereas our entire cohort received concurrent CRT, the standard of care in North America. Furthermore, $33.1 \%$ of patients in this study had squamous cell carcinoma (SCC), greater than the $17.3 \%$ we report in our cohort.

A few meta-analyses have investigated the association of sarcopenia and long-term outcomes in patients with esophageal or gastrointestinal malignancy $(8,9,14)$. A metaanalysis of 11 studies that reported the results of patients with esophageal cancer who underwent esophagectomy found those with sarcopenia had worse 3- and 5-year survival than those without sarcopenia (14). However, only 1 out of the 11 studies included was from the United States, with a majority of the studies included coming from Asia. Another meta-analysis examining the outcomes of patients with gastrointestinal malignancies, which included some esophageal cancer patients, found those with sarcopenia had worse long-term outcomes than those without sarcopenia who underwent esophagectomy (9). This analysis also included only 1 study from the United States, thus it is unclear if these results are applicable to North American cohorts.

It has been suggested that preoperative interventions 
can be included in enhanced recovery after surgery (ERAS) protocols to improve outcomes of sarcopenic patients who undergo combined CRT and esophagectomy (7). The concept of optimizing functional and nutritional status prior to major operations is not new, often being termed prehabilitation (30). A randomized control trial recently investigated the implementation of a prehabilitation program, including both preoperative exercise and nutritional therapy, in a cohort of patients with esophageal cancer (7). They found that those randomized to the prehabilitation program had improved pre- and postoperative functional capacity compared to those in the control group. Another study reported that a prehabilitation program improved outcomes in patients with esophageal cancer that underwent induction CRT (31). The authors reported that their intervention led to less functional decline after chemotherapy and less need for intravenous nutritional support. Although these studies are promising, the impact of prehabilitation programs on the short- and long-term outcomes of patients with LAEC who undergo esophagectomy is still unclear and requires further investigation.

There a number of reports that have examined the association of preoperative sarcopenia and development of postoperative complications after esophagectomy. Patients with sarcopenia in the immediate preoperative period have been found to be prone to respiratory complications after esophagectomy including re-intubation, respiratory failure, and pneumonia $(14,21,32)$. Among our cohort, 9 patients within the sarcopenia group developed respiratory complications, secondary to pneumonia, reintubation, and post-operative respiratory failure versus only 1 patient in the non-sarcopenia group who developed post-operative respiratory failure. Although this finding was not statistically significant the current study was inadequately powered to assess this outcome.

There are certain limitations to this study. First, we examined a relatively small cohort of patients from a single institution that underwent combined induction CRT and esophagectomy for esophageal cancer, potentially limiting external validity. In addition, although the cohort was similar in regard to baseline demographics and therapy received, there was some degree of diversity in therapeutic regimens and surgical techniques that may have introduced bias. Finally, 7 patients had to be excluded for being lost to follow-up which could potentially confound survival analysis results.

The current study suggests that preoperative sarcopenia in patients with LAEC at a single institution who received induction CRT is associated with worse OS and DFS after esophagectomy. Reports within the western hemisphere are currently conflicted on the impact sarcopenia has on the outcomes of patients who undergo esophagectomy. Patients found to be sarcopenic in the preoperative period may require more aggressive physical and nutritional optimization to mitigate these findings. However, this is yet to be confirmed in a prospective study and further research is needed to determine whether prehabilitation programs and enhanced ERAS protocols can improve the poor outcomes seen in this patient population.

\section{Acknowledgments}

An abstract of this work was presented at the 2020 American College of Surgeons Clinical Congress Scientific Forum. Funding: None.

\section{Footnote}

Reporting Checklist: The authors have completed the STROBE reporting checklist. Available at http://dx.doi. org/10.21037/jtd-20-2608

Peer Review File: Available at http://dx.doi.org/10.21037/jtd20-2608

Data Sharing Statement: Available at http://dx.doi. org/10.21037/jtd-20-2608

Conflicts of Interest: All authors have completed the ICMJE uniform disclosure form (available at http://dx.doi. org/10.21037/jtd-20-2608). CWS serves as an unpaid editorial board member of Fournal of Thoracic Disease from Jul 2017 to Jun 2021. The other authors have no conflicts of interest to declare.

Ethical Statement: The authors are accountable for all aspects of the work in ensuring that questions related to the accuracy or integrity of any part of the work are appropriately investigated and resolved. The study was conducted in accordance with the Declaration of Helsinki (as revised in 2013) and the Harmonized Tripartite Guideline for Good Clinical Practice from the International Conference on Harmonization. This study was reviewed and approved by the Rush University Medical Center Institutional Review Ethics Board (approval number 
19100703-IRB01) and since it was retrospective in nature a waiver of the requirement for informed consent was included with approval.

Open Access Statement: This is an Open Access article distributed in accordance with the Creative Commons Attribution-NonCommercial-NoDerivs 4.0 International License (CC BY-NC-ND 4.0), which permits the noncommercial replication and distribution of the article with the strict proviso that no changes or edits are made and the original work is properly cited (including links to both the formal publication through the relevant DOI and the license). See: https://creativecommons.org/licenses/by-nc-nd/4.0/.

\section{References}

1. Enzinger PC, Mayer RJ. Esophageal cancer. N Engl J Med 2003;349:2241-52.

2. Pennathur A, Gibson MK, Jobe BA, et al. Oesophageal carcinoma. Lancet 2013;381:400-12.

3. Wheeler JB, Reed CE. Epidemiology of esophageal cancer. Surg Clin North Am 2012;92:1077-87.

4. Domper Arnal MJ, Ferrandez Arenas A, Lanas Arbeloa A. Esophageal cancer: Risk factors, screening and endoscopic treatment in Western and Eastern countries. World J Gastroenterol 2015;21:7933-43.

5. Bailey SH, Bull DA, Harpole DH, et al. Outcomes after esophagectomy: a ten-year prospective cohort. Ann Thorac Surg 2003;75:217-22; discussion 22.

6. Orringer MB, Marshall B, Chang AC, et al. Two thousand transhiatal esophagectomies: changing trends, lessons learned. Ann Surg 2007;246:363-72; discussion 72-4.

7. Minnella EM, Awasthi R, Loiselle SE, et al. Effect of Exercise and Nutrition Prehabilitation on Functional Capacity in Esophagogastric Cancer Surgery: A Randomized Clinical Trial. JAMA Surg 2018;153:1081-9.

8. Joglekar S, Nau PN, Mezhir JJ. The impact of sarcopenia on survival and complications in surgical oncology: A review of the current literature--Author response. J Surg Oncol 2015;112:910.

9. Su H, Ruan J, Chen T, et al. CT-assessed sarcopenia is a predictive factor for both long-term and shortterm outcomes in gastrointestinal oncology patients: a systematic review and meta-analysis. Cancer Imaging 2019;19:82.

10. Ando N, Kato H, Igaki H, et al. A randomized trial comparing postoperative adjuvant chemotherapy with cisplatin and 5-fluorouracil versus preoperative chemotherapy for localized advanced squamous cell carcinoma of the thoracic esophagus. Ann Surg Oncol 2012;19:68-74.

11. van Hagen P, Hulshof MCCM, van Lanschot JJB, et al. Preoperative chemoradiotherapy for esophageal or junctional cancer. N Engl J Med 2012;366:2074-84.

12. Martin L, Birdsell L, Macdonald N, et al. Cancer cachexia in the age of obesity: skeletal muscle depletion is a powerful prognostic factor, independent of body mass index. J Clin Oncol 2013;31:1539-47.

13. Siegal SR, Dolan JP, Dewey EN, et al. Sarcopenia is not associated with morbidity, mortality, or recurrence after esophagectomy for cancer. Am J Surg 2018;215:813-7.

14. Deng HY, Zha P, Peng L, et al. Preoperative sarcopenia is a predictor of poor prognosis of esophageal cancer after esophagectomy: a comprehensive systematic review and meta-analysis. Dis Esophagus 2019;32:doy115.

15. Paireder M, Asari R, Kristo I, et al. Impact of sarcopenia on outcome in patients with esophageal resection following neoadjuvant chemotherapy for esophageal cancer. Eur J Surg Oncol 2017;43:478-84.

16. Elliott JA, Doyle SL, Murphy CF, et al. Sarcopenia: Prevalence, and Impact on Operative and Oncologic Outcomes in the Multimodal Management of Locally Advanced Esophageal Cancer. Ann Surg 2017;266:822-30.

17. Black D, Mackay C, Ramsay G, et al. Prognostic Value of Computed Tomography: Measured Parameters of Body Composition in Primary Operable Gastrointestinal Cancers. Ann Surg Oncol 2017;24:2241-51.

18. Grotenhuis BA, Shapiro J, van Adrichem S, et al. Sarcopenia/Muscle Mass is not a Prognostic Factor for Short- and Long-Term Outcome After Esophagectomy for Cancer. World J Surg 2016;40:2698-704.

19. Anandavadivelan P, Brismar TB, Nilsson M, et al. Sarcopenic obesity: A probable risk factor for dose limiting toxicity during neo-adjuvant chemotherapy in oesophageal cancer patients. Clin Nutr 2016;35:724-30.

20. Yip C, Goh V, Davies A, et al. Assessment of sarcopenia and changes in body composition after neoadjuvant chemotherapy and associations with clinical outcomes in oesophageal cancer. Eur Radiol 2014;24:998-1005.

21. Nakashima Y, Saeki H, Nakanishi R, et al. Assessment of Sarcopenia as a Predictor of Poor Outcomes After Esophagectomy in Elderly Patients With Esophageal Cancer. Ann Surg 2018;267:1100-4.

22. Tamandl D, Paireder M, Asari R, et al. Markers of sarcopenia quantified by computed tomography predict adverse long-term outcome in patients with resected 
oesophageal or gastro-oesophageal junction cancer. Eur Radiol 2016;26:1359-67.

23. Harada K, Ida S, Baba Y, et al. Prognostic and clinical impact of sarcopenia in esophageal squamous cell carcinoma. Dis Esophagus 2016;29:627-33.

24. Tan BH, Brammer K, Randhawa N, et al. Sarcopenia is associated with toxicity in patients undergoing neoadjuvant chemotherapy for oesophago-gastric cancer. Eur J Surg Oncol 2015;41:333-8.

25. Prado CM, Lieffers JR, McCargar LJ, et al. Prevalence and clinical implications of sarcopenic obesity in patients with solid tumours of the respiratory and gastrointestinal tracts: a population-based study. Lancet Oncol 2008;9:62935 .

26. Park SY, Yoon JK, Lee SJ, et al. Prognostic value of preoperative total psoas muscle area on long-term outcome in surgically treated oesophageal cancer patients. Interact Cardiovasc Thorac Surg 2017;24:13-9.

27. Makiura D, Ono R, Inoue J, et al. Impact of Sarcopenia on Unplanned Readmission and Survival After Esophagectomy in Patients with Esophageal Cancer. Ann

Cite this article as: Wakefield CJ, Hamati F, Karush JM, Arndt AT, Geissen N, Liptay MJ, Borgia JA, Basu S, Seder CW. Sarcopenia after induction therapy is associated with reduced survival in patients undergoing esophagectomy for locallyadvanced esophageal cancer. J Thorac Dis 2021;13(2):861-869. doi: $10.21037 /$ jtd-20-2608
Surg Oncol 2018;25:456-64.

28. Sheetz KH, Zhao L, Holcombe SA, et al. Decreased core muscle size is associated with worse patient survival following esophagectomy for cancer. Dis Esophagus 2013;26:716-22.

29. Rutten IJG, Ubachs J, Kruitwagen RFPM, et al. Psoas muscle area is not representative of total skeletal muscle area in the assessment of sarcopenia in ovarian cancer. J Cachexia Sarcopenia Muscle 2017; 8:630-8.

30. Carli F, Zavorsky GS. Optimizing functional exercise capacity in the elderly surgical population. Curr Opin Clin Nutr Metab Care 2005;8:23-32.

31. Xu YJ, Cheng JC, Lee JM, et al. A Walk-andEat Intervention Improves Outcomes for Patients With Esophageal Cancer Undergoing Neoadjuvant Chemoradiotherapy. Oncologist 2015;20:1216-22.

32. Ida S, Watanabe M, Yoshida N, et al. Sarcopenia is a Predictor of Postoperative Respiratory Complications in Patients with Esophageal Cancer. Ann Surg Oncol 2015;22:4432-7. 